\title{
Anatomic MR Imaging and Functional Diffusion Tensor Imaging of Peripheral Nerve Tumors and Tumorlike Conditions
}

\author{
A. Chhabra, R.S. Thakkar, G. Andreisek, M. Chalian, A.J. Belzberg, J. Blakeley, A. Hoke, G.K. Thawait, J. Eng, and J.A. Carrino
}

\begin{abstract}
BACKGROUND AND PURPOSE: A number of benign and malignant peripheral nerve tumor and tumorlike conditions produce similar imaging features on conventional anatomic MR imaging. Functional MR imaging using DTI can increment the diagnostic performance in differentiation of these lesions. Our aim was to evaluate the role of 3 T anatomic MR imaging and DTI in the characterization of peripheral nerve tumor and tumorlike conditions.
\end{abstract}

MATERIALS AND METHODS: Twenty-nine patients (13 men, 16 women; mean age, $41 \pm 18$ years; range, $11-83$ years) with a nerve tumor or tumorlike condition (25 benign, 5 malignant) underwent 3T MR imaging by using anatomic $(n=29)$, functional diffusion, DWI ( $n=21)$, and $\mathrm{DTI}(n=24)$ techniques. Images were evaluated for image quality (3-point scale), ADC of the lesion, tractography, and fractional anisotropy of nerves with interobserver reliability in ADC and FA measurements.

RESULTS: No significant differences were observed in age (benign, $40 \pm 18$ versus malignant, $45 \pm 19$ years) and sex (benign, male/female $=$ 12:12 versus malignant, male/female $=3: 2)(P>.05)$. All anatomic (29/29, 100\%) MR imaging studies received "good" quality; 20/21 (95\%) DWI and 21/24 (79\%) DTI studies received "good" quality. ADC of benign lesions $\left(1.848 \pm 0.40 \times 10^{-3} \mathrm{~mm}^{2} / \mathrm{s}\right)$ differed from that of malignant lesions $\left(0.900 \pm 0.25 \times 10^{-3} \mathrm{~mm}^{2} / \mathrm{s}, P<.001\right)$ with excellent interobserver reliability $(\mathrm{ICC}=0.988[95 \% \mathrm{Cl}, 0.976-0.994])$. There were no FA or ADC differences between men and women $(P>.05)$. FA of involved nerves was lower than that in contralateral healthy nerves $(P<$ $.001)$ with excellent interobserver reliability $(\mathrm{ICC}=0.970[95 \% \mathrm{Cl}, 0.946-0.991])$. ADC on DTI and DWI was not statistically different $(P>$ $.05)$, with excellent intermethod reliability $(\mathrm{ICC}=0.943[95 \% \mathrm{Cl}, 0.836-0.980])$. Tractography differences were observed in benign and malignant lesions.

CONCLUSIONS: 3T MR imaging and DTI are valuable methods for anatomic and functional evaluation of peripheral nerve lesions with excellent interobserver reliability. While tractography and low FA provide insight into neural integrity, low diffusivity values indicate malignancy in neural masses.

ABBREVIATIONS: BPNST = benign peripheral nerve sheath tumor; $\mathrm{Cl}=$ confidence interval; $\mathrm{CMT}=$ Charcot-Marie-Tooth disease; $\mathrm{FA}=$ fractional anisotropy; $\mathrm{ICC}=$ intraclass correlation coefficient; MPNST = malignant peripheral nerve sheath tumor

Cocal or diffuse enlargement of the peripheral nerves can be caused by tumors, such as benign and malignant peripheral nerve sheath tumors, perineurioma, lipoma, ganglion cyst, fibrolipomatous hamartoma and lymphoma, or hypertrophic neuropathy, such as Charcot-Marie-Tooth disease. ${ }^{1,2}$ Electrodiagnostic studies and clinical features can confirm neuropathy; however, high-resolution MR

Received June 22, 2012; accepted after revision July 19.

From the Russell H. Morgan Department of Radiology and Radiological Science (A.C., R.S.T., M.C., G.K.T., J.E., J.A.C.), and Departments of Neurosurgery (A.J.B.) and Neurology (J.B., A.H.), Johns Hopkins Hospital, Baltimore, Maryland; and Diagnostic and Interventional Radiology (G.A.), Zurich University Hospital, Zurich, Switzerland.

Please address correspondence to Avneesh Chhabra, MD, Russell H. Morgan Department of Radiology and Radiological Science, Johns Hopkins Hospital, $601 \mathrm{~N}$ Caroline St, Baltimore, MD 21287; e-mail: achhabr6@jhmi.edu

http://dx.doi.org/10.3174/ajnr.A3316 imaging of peripheral nerves (also referred to as MR neurography) is often needed to localize and characterize the causative mass. ${ }^{3-6} \mathrm{MR}$ imaging can be accomplished by using conventional (anatomic) techniques and diffusion-based (functional) techniques. 2D and 3D anatomic techniques are popular because they are not technically as challenging to perform as diffusion imaging. ${ }^{3-8}$ Lipoma, ganglion cyst, and fibrolipomatous hamartoma produce fairly diagnostic imaging features with these techniques. However, other lesions such as BPNST, MPNST, lymphoma, perineurioma, and hypertrophic neuropathy frequently show similar features with nonspecific T2 hyperintensity of nerves. Thus, differentiation of benign and malignant lesions can be difficult, even when suggested MR imaging features for malignancy, such as larger size, necrosis, hemorrhage, heterogeneous enhancement, and invasive margins, are present. Therefore, biopsy is often needed for definitive diagnosis. ${ }^{1,2,8-10}$ 
Diffusion-based MR neurography using DWI and DTI has the potential to overcome the limitations of anatomic MR imaging due to its ability to interrogate tissue microstructure. ${ }^{4,5,11}$ The unique fibrillar structure of the nerve results in anisotropy in the proton movement across its long axis, which can be mapped to create tracts and calculate DTI parameters, such as fractional anisotropy and apparent diffusion coefficient. In this original article, the authors evaluated both techniques with emphasis on diffusion imaging in the characterization of various peripheral nerve tumors and tumorlike conditions.

\section{MATERIALS AND METHODS}

\section{Study Subjects}

After institutional review board approval was granted, the records of our biweekly MR neurography conference (from October 2010 to April 2012) were reviewed, and 29 patients ( 13 men, 16 women; mean age, $41 \pm 18$ years; age range, $11-83$ years) with a nerve tumor or tumorlike condition ( 25 benign, 5 malignant) were included in those for whom the final diagnosis was established. The cases either had surgical proof ( 7 schwannomas, 2 neurofibromas, 1 degenerated schwannoma, 1 perineurioma, 3 MPNSTs, 1 lymphoma) and biopsy (6 schwannomas) or were diagnosed on the basis of clinical features, imaging characteristics, and stability up to 18-month follow-up, ${ }^{7}$ or had genetic testing (1 Charcot-MarieTooth disease type 1A). Two patients had undergone prior subtotal excision surgery and had a residual schwannoma. Two patients had underlying neurofibromatosis type 1 . The lesions were distributed as follows: brachial plexus $(n=15)$, lumbosacral plexus $(n=5)$, and peripheral nerves ( $n=10$ : three femoral, 1 sciatic, 1 radial, 2 elbow sensory, 1 axillary, 2 thoracic spinal). All patients presented with a variable combination of nonspecific regional symptoms of pain, weakness, and/or palpable mass. Mass lesions with pathognomonic appearances on anatomic MR imaging, such as internal fibrofatty proliferation (fibrolipomatous hamartoma), uniform fat (lipoma), and cystic lesion (ganglion cyst), were excluded.

\section{MR Imaging}

All imaging was performed on 3T MR imaging scanners (Magnetom Verio or Trio; Siemens, Erlangen, Germany) by using multichannel coil high-resolution 2D (T2 spectral-attenuated inversion recovery [SPAIR]: TR/TE/turbo factor [TF], 4500/80/17 ms; flip angle, $150^{\circ}$; section thickness, $4 \mathrm{~mm}$; averages, 2; base resolution, 704; in-plane resolution, $0.5 \mathrm{~mm}$ ) and $3 \mathrm{D}$ (short $\tau$ inversion recovery [STIR] and sampling perfection with application optimized contrasts by using different flip angle evolution [SPACE]: TR/TE/TF, 1500/103/61 ms; TI, $220 \mathrm{~ms}$; section thickness, 1.0 $\mathrm{mm}$; averages, 1.4; base resolution, 320; in-plane resolution, 1.0 $\mathrm{mm})$ imaging. All patients had similar anatomic imaging $(29 / 29$, $100 \%$ ), while $21 / 29(72 \%)$ had DWI (b values 50, 400, and 800 $\left.\mathrm{s} / \mathrm{mm}^{2}\right)$, and 24/29 (83\%) had DTI $\left(0,800,1000 \mathrm{~s} / \mathrm{mm}^{2} ; 14 / 24\right.$ with 12 directions and 10/24 with 20 directions of interrogation).

\section{Image Analysis}

Two radiologists (A.C. with 14 years and R.S.T. with 4 years of radiology experience) interpreted all MR imaging studies. They were blinded to the final diagnosis and used a dedicated workstation (Leonardo; Siemens).

First, all images were assessed for motion, ghosting artifacts, and incomplete inclusion of the lesion. On the basis of a combination of these findings, the quality of anatomic, DWI, and DTI scans was rated by using a 3-point scale as good (1), fair (2), and poor (3).

Second, the anatomic images were used for lesion localization and characterization and then were assessed in tandem with DWI and DTI to perform functional evaluation.

Third, the ADC of the lesion, particularly of the solid-appearing portion, was measured separately by both observers on DWI and DTI by manual placement of at least a 6- to 8-mm circular region of interest. The lowest $\mathrm{ADC}$ was recorded to encompass the greatest degree of restriction (cellularity).

Fourth, tensor calculation of the raw DTI and tractography was performed by using Neuro 3D syngo software (Siemens) by placing multiple seed points along the nerve. There is currently no standardization with regard to DTI evaluation, and the angle and FA thresholds were thus arbitrarily chosen, depending on the obliquity of the nerve and pathology. ${ }^{4,5,11}$ We chose an FA threshold (minimum value) of 0.2 , an angle threshold (maximum turning angle) of $45^{\circ}$, and a step length of $0.9 \mathrm{~mm}$ for all tractographies. Fiber tracts were calculated by using the fourth-order Runge-Kutta algorithm. The postprocessing procedure took approximately 20 minutes with a mean of $25 \pm 10$ minutes. The manual placing of seed points avoided contamination from spurious tracts from adjacent muscles or slow-flowing veins.

Fifth, all fiber tract images were evaluated by both readers in consensus to decide if the reconstructed fiber tracts showed a normal appearance (similar thickness to the contralateral nerve or adjacent normal nerves with no disruption/deviation), near-normal appearance (minimal variation in thickness with $<25 \%$ disruption of transverse fiber attenuation), partial discontinuity (moderate variation in thickness with 25\%-75\% disruption of transverse fiber attenuation), or near-complete/complete disruption (severe variation in thickness with $>75 \%$ disruption of transverse fiber attenuation).

Last, FA of the nerves was assessed separately by each observer independently by placing a user-defined 3- to 4-mm circular region of interest on the axial parameter maps of the nerve proximal to mass lesion, and this was compared with the contralateral nerve. Three measurements were obtained, and the lowest FA was recorded to assess the maximal loss of anisotropy (potential axonal degeneration/demyelination).

\section{Statistical Analysis}

All data were stored on a spreadsheet (Excel 2010; Microsoft, Seattle, Washington). Analysis was performed by using the Statistical Package for the Social Sciences software (Version 18.0; SPSS, Chicago, Illinois). A nonparametric Wilcoxon rank-sum test was used for side-to-side and also between-group comparisons of the quantitative variables. A $\chi^{2}$ or Fisher exact test was used for the categoric variables. A Pearson correlation was used to examine the correlation between ADC values on DTI and DWI. A probability $<.05$ was accepted as statistically significant. Interobserver and intraobserver reliability were assessed by using the intraclass cor- 


\begin{tabular}{|c|c|}
\hline Patient Information & Data \\
\hline $\begin{array}{l}\text { Demographics and imaging } \\
\text { characteristics }\end{array}$ & $\begin{array}{l}\text { No. }=29, \text { male } / \text { female }=13: 16, \text { mean age }=41 \pm 18 \mathrm{yr} \text {, range }=11-83 \mathrm{yr} ;{ }^{\mathrm{a}} \\
\text { left/right }=9: 21 \text { lesions } \\
\text { DTI ADC }=24 \text { patients, DWI ADC }=21 \text { patients } \\
\text { No. of directions in DTI: } 14 \text { patients with } 12,10 \text { patients with } 20 \text { directions, respectively }\end{array}$ \\
\hline Benign and malignant lesions & $\begin{array}{l}\text { Benign }=25 \text {, malignant }=5 \\
\text { No difference in terms of age (benign }=40 \pm 18 \text { versus malignant }=45 \pm 19 \mathrm{yr} \text { ), sex (benign, } \\
\quad \text { male/female }=12: 13 \text { versus malignant, male/female }=3: 2)(P>.05) \text {, FA and ADC values }(P>0.05) ;{ }^{a} \\
\text { DTI ADC different }(P=.001) \text {, benign }=1.848 \pm 0.40 \text { versus malignant }=0.900 \pm 0.25 \times 10^{-3} \mathrm{~mm}^{2} / \mathrm{s} ;{ }^{a} \\
\text { DWI ADC not different }(P>.05) \text {, benign }=1.745 \pm 0.44 \text { vs malignant }=1.267 \pm 0.35 \times 10^{-3} \mathrm{~mm}^{2} / \mathrm{s}^{\mathrm{a}} \\
\text { Interobserver reliability ADC: ICC }=0.988(95 \% \mathrm{Cl}, 0.976-0.994) \text {, excellent }{ }^{\mathrm{a}}\end{array}$ \\
\hline $\begin{array}{l}\text { Abnormal and contralateral normal } \\
\text { nerves }\end{array}$ & $\begin{array}{l}\text { FA significantly different }(P<.001) \text {, abnormal }=0.221 \pm 0.09 \text {, normal }=0.344 \pm 0.12 \text {; }^{\mathrm{a}} \\
\text { Interobserver reliability } \mathrm{FA}: \mathrm{ICC}=0.970(95 \% \mathrm{Cl}, 0.946-0.991) \text {, excellent }{ }^{\mathrm{a}}\end{array}$ \\
\hline $\begin{array}{l}\text { DTI ADC among benign lesions betw } \\
\text { different ( } 12 \text { and } 20) \text { directions }\end{array}$ & $\begin{array}{l}\text { nNot statistically different }(P>.05) \\
\text { DTI ADC on } 12 \text { directions: } 1.627 \pm 0.600 \\
\text { DTI ADC on } 20 \text { directions: } 1.520 \pm 0.390\end{array}$ \\
\hline DTI ADC and DWI ADC & $\begin{array}{l}\text { Not statistically different }(P>.05) \\
\mathrm{ICC}=0.943(95 \% \mathrm{Cl}, 0.836-0.980) \text {, excellent intermethod reliability } \\
\text { Very good Pearson correlation }(r=0.90, P<.001)\end{array}$ \\
\hline
\end{tabular}

a years.

relation coefficient $(95 \% \mathrm{CI})$. Scores were interpreted on the basis of the values suggested by Shrout and Fleiss ${ }^{12}$ with a score of $0-0.4$ indicating poor reliability, $0.4-0.75$ indicating moderate reliability, and $>0.75$ indicating excellent reliability.

\section{RESULTS}

Demographics, Image Quality, and Anatomic Appearance There were no significant differences in terms of age (benign, $40 \pm 18$ versus malignant, $45 \pm 19$ years) and sex (benign, male/ female $=12: 12$ versus malignant, male/female $=3: 2$ ) $(P>.05)$. All anatomic $(29 / 29,100 \%)$ MR imaging received a "good" quality, while 20/21 (95\%) DWI and 19/24 (79\%) DTI studies received a "good" quality. Three of 24 (13\%) DTI studies received "fair" quality due to suboptimal SNR. Two of $24(8 \%)$ DTI studies received a "poor" quality rating because no meaningful tracts could be generated due to incomplete lesion inclusion and ghosting artifacts. One of 21 (4\%) DWI studies received fair quality. All masses were apparent on both anatomic imaging and DTI as hyperintense lesions. The lesions showed variable shapes (4 rounded, 15 oval, and 11 elongated) and heterogeneity (18/30, $60 \%$ ). The lesions measured $5.94 \pm 6.45 \mathrm{~mm}$ (range, $0.8-34.7$ $\mathrm{mm}$ ). None of the malignant lesions showed satellite lesions, local invasion, or distant metastases.

\section{ADC and FA}

Detailed results are shown in the Table. The ADC of benign lesions $\left(1.848 \pm 0.40 \times 10^{-3} \mathrm{~mm}^{2} / \mathrm{s}\right)$ differed from that of malignant lesions $\left(0.900 \pm 0.25 \times 10^{-3} \mathrm{~mm}^{2} / \mathrm{s} ; P<.001\right)$ with excellent interobserver reliability (ICC $=0.988$ [95\% CI, 0.9760.994]). There were no ADC or FA differences between men and women $(P>.05)$. The FA of involved nerves was lower than that of contralateral nerves $(P<.001)$ with excellent interobserver reliability (ICC $=0.970$ [95\% CI, 0.946-0.991]). ADC measured on DTI and DWI techniques in the same patients $(n=16)$ was not statistically different $(P>.05)$, with a very good Pearson correlation $(r=0.90, P<.001)$ and excellent intermethod reliability $(\mathrm{ICC}=0.943$ [95\% CI, 0.836-0.980]) (Fig 1). However, DWI ADC was not statistically different $(P>.05$; benign, $1.745 \pm 0.44$

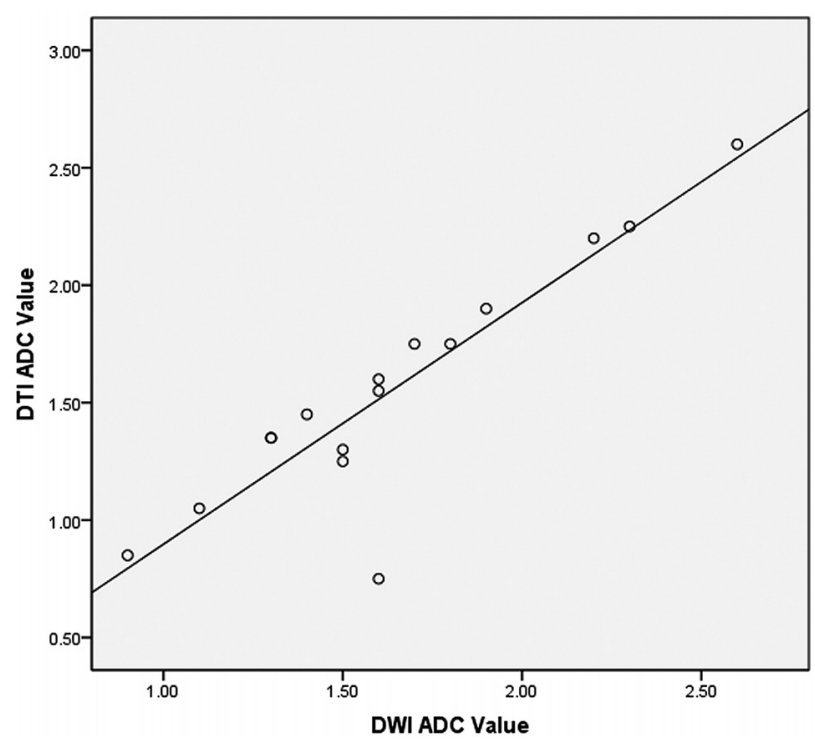

FIG 1. ADC on DWI and DTI. There is a very good Pearson correlation $(r=0.90, P<.001)$.

versus malignant, $1.267 \pm 0.35)$. Additionally, among the benign lesions, ADC (1.627 \pm 0.600$)$ in 12-direction DTI was not statistically different from 20 -direction DTI $(1.520 \pm 0.390, P>.05)$.

\section{Fiber Tractography}

On tractography, no lesion had a normal appearance of the tract. Complete disruption was observed in 4 lesions: 1 degenerated schwannoma, 1 plexiform neurofibroma, and 2 MPNSTs. Partial disruption was seen in 8 lesions: 1 MPNST, 2 schwannomas, 3 BPNSTs, and 2 residual schwannomas (Figs 2 and 3). Near-normal tracts in the background of nerve swelling were seen in 8 lesions: 1 lymphoma, 1 perineurioma, 2 (bilateral) nerves of CMT, 2 schwannomas, and 2 BPNSTs, (Figs 4 and 5). Due to oblique course of the nerves, the Neuro 3D syngo software did not reproduce color coding similar to the traditional red, green, blue colors used in brain tractography. Five of 25 (20\%) lesions could not be traced due to suboptimal SNR/ghosting artifacts, and no meaningful tracts could be generated from those lesions. 

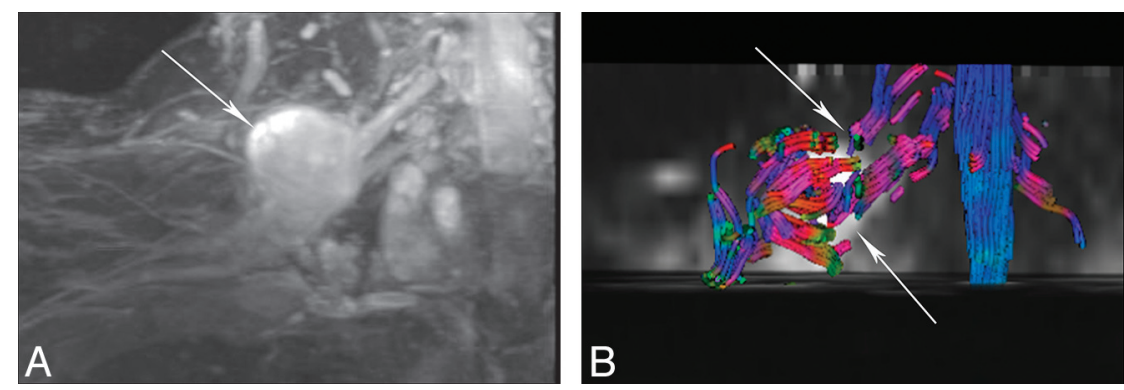

FIG 2. A, MPNST. 3D STIR SPACE maximum-intensity-projection image in 56-year-old man presenting with a painless palpable mass in the right supraclavicular region for 6 months, which was steadily increasing in size with progressive weakness of right upper extremity. The mass involves the C5 and C6 nerve roots and upper trunk (arrow). B, Corresponding DTI shows disorganization and partial (near-complete) disruption of tracts (arrows). The ADC is $0.9 \times 10^{-3} \mathrm{~mm}^{2} / \mathrm{s}$.
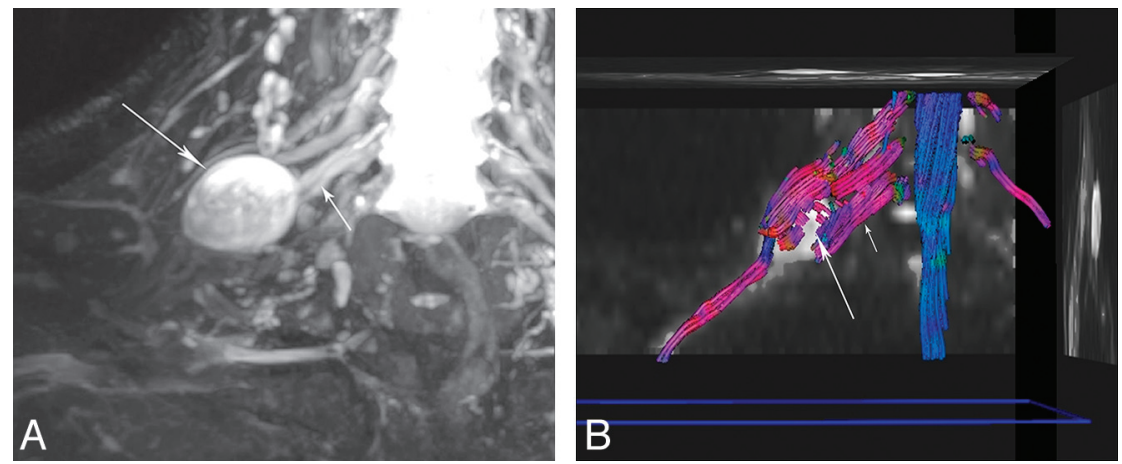

FIG 3. A, BPNST. 3D STIR SPACE maximum-intensity-projection image in a 34-year-old man presenting with a painless palpable mass in the right supraclavicular region. The mass remained stable during a 12-month period. The mass (large arrow) involves the distal right C7 nerve root (small arrow) and the upper trunk of the brachial plexus. B, Corresponding DTI shows swelling of the right C7 nerve root (small arrow) with partial disruption of the tracts (large arrow). The ADC is $1.7 \times 10^{-3} \mathrm{~mm}^{2} / \mathrm{s}$.
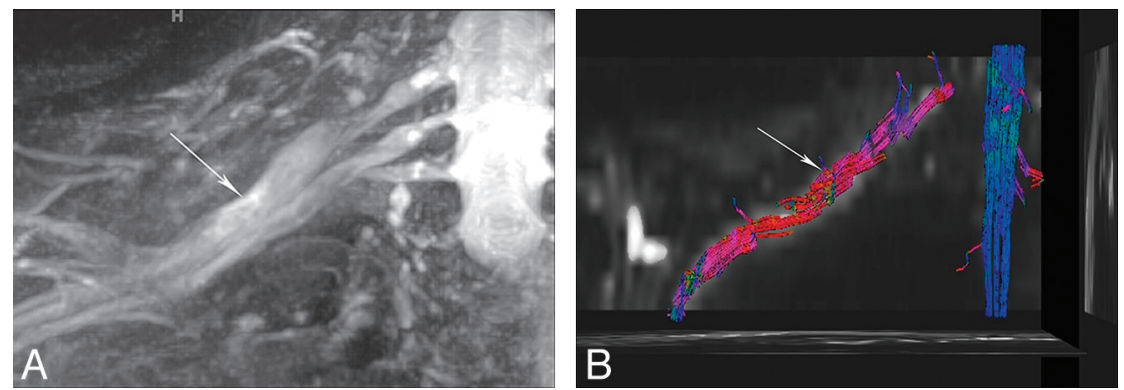

FIG 4. A, Lymphoma. 3D STIR SPACE maximum-intensity-projection image in 65-year-old man presenting with progressively increasing weakness of the right arm during a few months. There was enlargement and abnormal hyperintense signal of multiple right brachial plexus nerve roots and trunks. The C6 nerve was the most abnormal (arrow). B, Corresponding DTI shows near-normal tracts. The ADC is $0.7 \times 10^{-3} \mathrm{~mm}^{2} / \mathrm{s}$
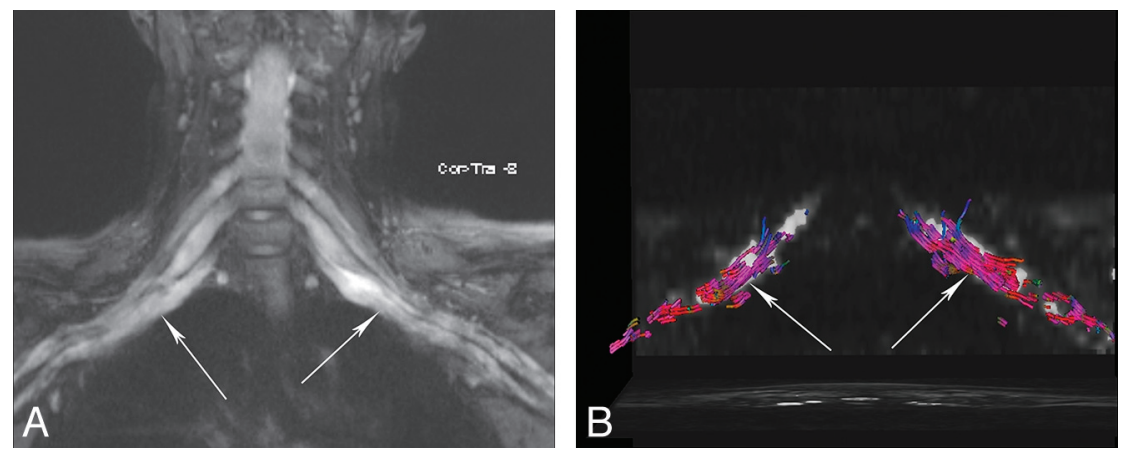

FIG 5. $A, C M T 1 A$ disease. 3D STIR SPACE maximum-intensity-projection image in a 28-year-old woman presenting with pain in the lower midline thoracic region for many months. A diffuse symmetrically enlarged brachial plexus was seen bilaterally (arrows). B, Corresponding DTI shows near-normal tracts (arrows), given the limitation of suboptimal SNR near the spine and peripheral areas. The ADC was 2.0 and $1.9 \times 10^{-3} \mathrm{~mm}^{2} / \mathrm{s}$ on right and left sides, respectively. 


\section{DISCUSSION}

The peripheral nerves may be affected by a variety of nerve tumors and tumorlike conditions. ${ }^{1,2,8-10}$ Anatomic 2D and 3D MR imaging consistently produced high-quality images and nicely depicted mass lesions along the long axis of the nerves and demonstrated associated nerve abnormalities. ${ }^{3-5}$ High-resolution 3D STIR SPACE images use variable flip angles, are relatively insensitive to magnetization transfer-related cross-talk among sections, and provide the best fat suppression for plexus evaluation. ${ }^{4}$ The images generated could then be matched to DTI for anatomic correlation and measurement of diffusion parameters. However, as seen in this study, the differentiation into benign or malignant nerve tumors was not possible due to similar appearances on anatomic imaging. Fluorine 18 FDG-PET has been found to be useful in this domain, though the standard uptake values among benign and malignant lesions overlap and the patient will have to undergo another examination apart from MR imaging for presurgical planning. ${ }^{13-15}$

DTI also produced good-quality images in most cases, despite prior surgery in the regional area in 2 cases. As opposed to standard diffusion imaging, DTI not only provides quantitative information about the degree and direction of water diffusion within the nerves but can also be used for tractography to visualize the orientation of axons/nerve tracts and to assess their integrity. ${ }^{16,17}$ We found ADC on DTI to be more useful than DWI in differentiating benign from malignant lesions; this result may be partly related to a larger number of directions in diffusion encoding and higher b-values used in our DTI technique $\left(1000 \mathrm{~s} / \mathrm{mm}^{2}\right.$ versus $800 \mathrm{~s} / \mathrm{mm}^{2}$ ). However, 12- versus -20 directions of interrogation did not make much difference in ADC evaluation. The findings of increasing ADC suggesting benignity of lesions are similar to those seen with other tumors, such as in the breast and prostate. ${ }^{18,19}$ Due to excellent interobserver reliability, apart from this valuable information, the ADC can also be potentially followed as a biomarker to detect tumor response/necrosis if the lesions are being followed longitudinally or after adjuvant medical treatment. Such scenarios are likely as new medical therapies (imatinib and other chemotherapies) are being developed and tested for schwannomatosis, MPNST, and lymphoma.

Due to cell membrane and myelin sheath barriers produced by tightly packed and coherently aligned axons, proton diffusion across the fibrillar structure of the nerve is slower than that along the long axis of the nerve, leading to higher radial anisotropy in normal nerves. ${ }^{16,17,20}$ The peripheral nerve ADC and FA vary depending on the magnet, coil, and technique; the nerve in question; age; and underlying comorbidities. Although the normal FA of peripheral nerves ranges between 0.3 and 0.7 , the side-to-side variations are minimal; the (healthy) contralateral nerve can serve as internal reference. ${ }^{21-25}$ The FA of the involved nerves was significantly lower than that of the contralateral nerves, likely reflecting associated axonal degeneration and myelin loss, similar to the results of Morisaki et al, ${ }^{22}$ Lehmann et al, ${ }^{23}$ and Khalil et al. ${ }^{24}$ Asymmetric T2 signal abnormality is a useful sign of segmental brachial plexus abnormality ${ }^{26}$; however, magic angle artifacts are also a known cause of this finding. DTI offers a quantitative tool with excellent interobserver reliability to document such an abnormality by showing lowering of FA, thereby confirming neu- ropathy. This was seen in the case of CMT, where bilateral low FA values were observed.

The fiber orientation information yielded by DTI provides a new contrast mechanism that can be used to resolve images of anatomic substructures. The predominant paths of proton movement determined by the principal eigenvector in each voxel can be mapped to create an anisotropy image using multiple directions of interrogation. ${ }^{27}$ The larger the number of directions used, the more robust the data can be. However, SNR may, accordingly, drop, and the acquisition time might be prolonged. Authors use 12-20 directions, depending on the software available on different 3T MR imaging scanners. DTI can be degraded by motion, susceptibility, and ghosting artifacts. ${ }^{27,28}$ In the authors' experience, using tighter echo spacing $(<0.07 \mathrm{~ms})$, parallel imaging, higher bandwidth, imaging in the axial plane, and good fat suppression (water-selective technique) mitigates many of these artifacts and increases SNR. The improvement in SNR at 3T helps in the creation of highly resolved DTI parametric maps with enhanced depiction of tracts and better coregistration with the expected anatomy on MR neurography images. ${ }^{18,28}$

In this study, tractography was possible in most lesions and was able to assess the microstructure of the nerves and associated lesions. Most benign lesions showed partial tract disruption or near-normal appearance with the exception of a degenerated schwannoma and a plexiform neurofibroma, which revealed complete tract disruption. We did not observe an isolated course deviation as a sign of BPNST as reported in a feasibility study by Vargas et al. ${ }^{5}$ This is likely explained by the fact that DTI is a functional technique and not direct anatomic imaging; therefore, areas of axonal degeneration and/or myelin loss will result in local loss of fiber attenuation, while anatomic fascicular architecture may still be present. Partial and complete disruption of tracts was noted in MPNSTs, which was also confirmed surgically. The nearnormal appearance of the tracts was also seen in lymphoma, CMT, and perineurioma; which are explainable on the basis of the permeative nature of lymphoma, predominant demyelination in CMT type $1 \mathrm{~A}$, and perineurial cell proliferation around thinly myelinated axons. ${ }^{29-31}$

Although we included the lesions with good reference standards, our limitations include lack of healthy controls. However, we used normal contralateral nerves as a control and noted significant side-to-side differences in FA. Despite the rarity of the nerve tumors and tumorlike conditions, we tried to encompass most of the lesions that one may encounter in routine practice and used a uniform technique on high-field imaging. In the future, larger prospective or multicenter trials by using DTI can further validate these findings.

\section{CONCLUSIONS}

Anatomic MR imaging and functional DTI are valuable and reliable methods for the noninvasive evaluation of peripheral nerve tumors. While tractography provides insight into neural integrity, low diffusivity values indicate malignancy in neural masses. Thus, DTI with fiber tracking has the potential to provide clinically relevant information that is beyond standard MR imaging techniques for the diagnosis and follow-up of nerve tumor and tumorlike conditions. 


\section{ACKNOWLEDGMENTS}

The authors acknowledge Aaron Flammang, Siemens Medical Systems, for technical help, and Vibhor Wadhwa, medical student, for assistance in the data collection.

Disclosures: Avneesh Chhabra—UNRELATED: Consultancy: Siemens, Comments: CAD system development, Grants/Grants Pending: Siemens Medical Solutions, ${ }^{*}$ Integra Life Sciences, ${ }^{*}$ GE-AUR, ${ }^{*}$ Travel/Accommodations/Meeting Expenses Unrelated to Activities Listed: Siemens, Integra, GE-AUR. Allan J. Belzberg-UNRELATED: Grants/Grants Pending: Children's Tumor Foundation. * Jaishri Blakeley-UNRELATED: Grants/Grants Pending: Department of Defense grants pertinent to NF1, NF2, and schwannomatosis are currently under review, Travel/Accommodations/Meeting Expenses Unrelated to Activities Listed: travel to a meeting with Sanofi regarding a trial for glioblastoma. John A. Carrino-UNRELATED: Board Membership: GE Healthcare, Consultancy: Quality Medical Metrics, Medtronic, Vital Imaging, Grants/Grants Pending: Siemens, ${ }^{*}$ Carestream, ${ }^{*}$ Toshiba;* Payment for Lectures (including service on Speakers Bureaus): Siemens, Stock/Stock Options: Merge Healthcare. *Money paid to the institution.

\section{REFERENCES}

1. Ogose A, Hotta T, Morita T, et al. Tumors of peripheral nerves: correlation of symptoms, clinical signs, imaging features, and histologic diagnosis. Skeletal Radiol 1999;28:183-88

2. Chhabra A, Thawait GK, Andreisek G. Peripheral nerve and tumorlike conditions. In: Chhabra A, Andreisek G, eds. Magnetic Resonance Neurography. New Delhi, India: Jaypee Brothers Medical Publishers; 2012:113-32

3. Mallouhi A, Marik W, Prayer D, et al. 3T MR tomography of the brachial plexus: structural and microstructural evaluation. Eur J Radiol 2012;81:2231-45

4. Viallon M, Vargas MI, Jlassi $\mathrm{H}$, et al. High-resolution and functional magnetic resonance imaging of the brachial plexus using an isotropic 3D T2 STIR (short term inversion recovery) SPACE sequence and diffusion tensor imaging. Eur Radiol 2008;18:1018-23

5. Vargas MI, Viallon M, Nguyen D, et al. Diffusion tensor imaging (DTI) and tractography of the brachial plexus: feasibility and initial experience in neoplastic conditions. Neuroradiology 2010;52: 237-45

6. Chhabra A, Lee PP, Bizzell C, et al. 3 Tesla MR neurography: technique, interpretation, and pitfalls. Skeletal Radiol 2011;40:1249-60

7. Subhawong TK, Wang KC, Thawait SK, et al. High resolution imaging of tunnels by magnetic resonance neurography. Skeletal Radiol 2012;41:15-31

8. Thawait SK, Chaudhry V, Thawait GK, et al. High-resolution MR neurography of diffuse peripheral nerve lesions. AJNR Am J Neuroradiol 2011;32:1365-72

9. Chhabra A, Soldatos T, Durand DJ, et al. The role of magnetic resonance imaging in the diagnostic evaluation of malignant peripheral nerve sheath tumors. Indian J Cancer 2011;48:328-34

10. Wasa J, Nishida Y, Tsukushi S, et al. MRI features in the differentiation of malignant peripheral nerve sheath tumors and neurofibromas. AJR Am J Roentgenol 194:1568-74

11. Khalil C, Budzik JF, Kermarrec E, et al. Tractography of peripheral nerves and skeletal muscles. Eur J Radiol 2010;76:391-97

12. Shrout PE, Fleiss JL. Intraclass correlations: uses in assessing rater reliability. Psychol Bull 1979;86:420-28
13. Shahid KR, Amrami KK, Esther RJ, et al. False-negative fluorine-18 fluorodeoxyglucose positron emission tomography of a malignant peripheral nerve sheath tumor arising from a plexiform neurofibroma in the setting of neurofibromatosis type 1. J Surg Orthop Adv 2011;20:132-35

14. Ohno T, Ogata K, Kogure N, et al. Gastric schwannomas show an obviously increased fluorodeoxyglucose uptake in positron emission tomography: report of two cases. Surg Today 2011;41:1133-37

15. Bertagna F, Bosio G, Biasiotto G, et al. Malignant transformation to schwannoma in a patient affected by type 1 neurofibromatosis as demonstrated by F-18-FDG-PET/CT. Nucl Med Rev Cent East Eur 2010;13:15-17

16. Tagliafico A, Calabrese M, Puntoni M, et al. Brachial plexus MR imaging: accuracy and reproducibility of DTI-derived measurements and fibre tractography at 3.0-T. Eur Radiol 2011;21:1764-71

17. Meek MF, Stenekes MW, Hoogduin HM, et al. In vivo three-dimensional reconstruction of human median nerves by diffusion tensor imaging. Exp Neurol 2006;198:479-82

18. Baltzer PA, Schäfer A, Dietzel M, et al. Diffusion tensor magnetic resonance imaging of the breast: a pilot study. Eur Radiol 2011;21: $1-10$

19. Oto A, Yang C, Kayhan A, et al. Diffusion-weighted and dynamic contrast-enhanced MRI of prostate cancer: correlation of quantitative MR parameters with Gleason score and tumor angiogenesis. AJR Am J Roentgenol 2011;197:1382-90

20. Chhabra A, Andreisek G, Soldatos T, et al. MR neurography: past, present, and future. AJR Am J Roentgenol 2011;197:583-91

21. Andreisek G, White LM, Kassner A, et al. Diffusion tensor imaging and fiber tractography of the median nerve at 1.5T: optimization of b value. Skeletal Radiol 2009;38:51-59

22. Morisaki S, Kawai $Y$, Umeda M, et al. In vivo assessment of peripheral nerve regeneration by diffusion tensor imaging. J Magn Reson Imaging 2011;33:535-42

23. Lehmann HC, Zhang J, Mori S, et al. Diffusion tensor imaging to assess axonal regeneration in peripheral nerves. Exp Neurol 2010; 223:238-44

24. Khalil C, Hancart C, Le Thuc V, et al. Diffusion tensor imaging and tractography of the median nerve in carpal tunnel syndrome-preliminary results. Eur Radiol 2008;18:2283-91

25. Sheikh KA. Non-invasive imaging of nerve regeneration. Exp Neurol 2010;223:72-76

26. Filler AG, Maravilla KR, Tsuruda JS. MR neurography and muscle MR imaging for image diagnosis of disorders affecting the peripheral nerves and musculature. Neurol Clin 2004;22:643-82, vi-vii

27. Mori S, van Zijl PC. Fiber tracking: principles and strategies-a technical review. NMR Biomed 2002;15:468-80

28. Okada T, Miki Y, Fushimi Y, et al. Diffusion-tensor fiber tractography: intraindividual comparison of 3.0-T and 1.5-T MR imaging. Radiology 2006;238:668-78

29. Skre H. Genetic and clinical aspects of Charcot-Marie-Tooth's disease. Clin Genet 1974;6:98-118

30. Reilly MM, Murphy SM, Laurá M. Charcot-Marie-Tooth disease. J Peripher Nerv Syst 2011;16:1-14

31. Mauermann ML, Amrami KK, Kuntz NL, et al. Longitudinal study of intraneural perineurioma: a benign, focal hypertrophic neuropathy of youth. Brain 2009;132(pt 8):2265-76 